\title{
Neovascularization Perfusion of Melolabial Flaps Using Intraoperative Indocyanine Green Angiography
}

Mohamed Abdelwahab, MD; Emily A. Spataro, MD; Cherian K. Kandathil, MD; Sam P. Most, MD

IMPORTANCE Assessment of melolabial flap perfusion using indocyanine green (ICG) angiography for nasal reconstruction has not been previously described.

OBJECTIVE To assess melolabial flap relative perfusion using ICG angiography in nasal reconstruction and highlight its clinical relevance.

DESIGN, SETTING, AND PARTICIPANTS In this retrospective case series at a tertiary referral center, 10 patients undergoing melolabial flap reconstruction of nasal defects between January 2015 to April 2018 with flap perfusion were assessed by ICG angiography.

EXPOSURES Indocyanine green angiography was performed to assess melolabial flap perfusion at second stage surgery after temporary clamping of the pedicle and after pedicle division and flap inset.

MAIN OUTCOMES AND MEASURES Flap perfusion in relation to a reference point on the cheek was calculated after both pedicle clamping and division by 2 methods: (1) ingress (arterial inflow) and egress (venous outflow) flap-to-cheek ratio; (2) flap-to-cheek perfusion (fluorescence) ratio at 3 time points (midpoint of ICG flap inflow, maximum [peak] fluorescence, and midpoint of ICG outflow), and their calculated mean. Correlations of perfusion with time between stages, and patient factors were performed; perfusion parameters after temporary pedicle clamping and after division and flap inset were compared.

RESULTS Ten patients (mean [SD] age, 66 [7.5] years) were enrolled with a mean (SD) interval of 23 (4) days between first and second surgery. No correlation existed between perfusion parameters and time interval between first and second stage, or any other patient factors. Of the different perfusion parameters, a statistically significant difference was observed when comparing temporary clamping and postdivision mean (SD) flap-to-cheek perfusion ratio (0.89 [0.22] and 0.80 [0.19]; $95 \% \mathrm{Cl}, 4.1-12.6 ; P=.001$ ), as well as mean (SD) peak perfusion ratio (0.81 [0.2] and 0.77 [0.2]; $95 \% \mathrm{Cl}, 0.005-0.080 ; P=.02)$ only. No partial or complete flap necrosis was reported after pedicle division.

CONCLUSIONS AND RELEVANCE Indocyanine green angiography is an effective method to determine neovascularization perfusion of melolabial flaps, with a mean perfusion of $89 \%$ after pedicle clamping. Future applications of this technology include use in patients at high risk for flap necrosis, or those who may benefit from early flap division to ensure adequate perfusion and minimize the time interval between staged procedures.

LEVEL OF EVIDENCE NA.
Author Affiliations: Division of Facial Plastic and Reconstructive Surgery, Department of Otolaryngology-Head \& Neck Surgery, Stanford University School of Medicine, Stanford, California (Abdelwahab, Spataro, Kandathil, Most); Department of Otolaryngology-Head \& Neck Surgery, Mansoura University Faculty of Medicine, Mansoura, Egypt (Abdelwahab).

Corresponding Author: Sam P. Most MD, Division of Facial Plastic and Reconstructive Surgery, Stanford University School of Medicine, 801 Welch Rd, Stanford, CA 94305 (smost@stanford.edu). 
$\mathrm{M}$ ohs surgery is one of the most common procedures performed in the United States for treating skin cancer of the face, and particularly the nose. The resulting defect requires precise reconstruction to achieve both an acceptable functional and aesthetic outcome. In large defects exceeding $1.5 \mathrm{~cm}$, skin grafts and local flaps generally do not provide adequate vascularized tissue, requiring the use of regional interpolated flaps. ${ }^{1,2}$ Forehead flaps are the workhorse of larger nasal reconstructions, however melolabial flaps are also very useful for reconstruction of lateral or lower third defects. ${ }^{3}$ Melolabial (nasolabial) flaps are random flaps, unlike forehead flaps, depending primarily on the perfusion pressure from the underlying subdermal plexus. The arterial blood supply arises from unnamed musculocutaneous arterial perforators supplied by the facial artery. ${ }^{4}$ These flaps often require division at a variable length of time after sufficient neovascularization takes place, with an average of 3 weeks between stages. ${ }^{4}$ Although inadequate perfusion of the distal flap after dividing the pedicle may result in partial or complete tissue loss, no studies have been conducted to assess the perfusion of melolabial flaps at the time of pedicle division.

Intraoperative flap perfusion has been assessed by several methods. These include handheld (pencil) doppler, ${ }^{5}$ color doppler (duplex) ultrasound, ${ }^{6}$ optical diffusion imaging spectroscopy, ${ }^{7}$ near-infrared spectroscopy, ${ }^{7,8}$ dynamic infrared thermography, ${ }^{9}$ fluorescein angiography, ${ }^{10}$ laser doppler flowmetry, ${ }^{11}$ and laser-assisted indocyanine green (ICG) angiography. ${ }^{12,13}$ Indocyanine green angiography using the SPY Elite Imaging System (NOVADAQ Technologies, Inc) has been used to objectively assess perfusion of paramedian forehead flaps in nasal reconstruction and is gaining popularity for its excellent safety, sensitivity, and specificity. ${ }^{14}$

The SPY Elite Imaging System consists of an 806-nm near-infrared laser and a high-resolution camera for nearinfrared imaging used in conjunction with ICG intravenous contrast allowing noninvasive angiography without radioactive contrast. Intravenously administered ICG binds completely to plasma proteins with a halflife of 3 to 4 minutes. ${ }^{15,16}$ Illumination of the area of interest by the 806-nm laser to the SPY Elite Imaging System causes the ICG dye to fluoresce. This process is captured by the camera and perfusion can be calculated as an absolute value or as a percentage relative to the predetermined reference point. ${ }^{12}$

The associated SPY-Q software is another advantage because it provides surgeons with a calculated relative perfusion. ${ }^{17-19}$ Indocyanine green angiography has been used to measure forehead flap perfusion at different time points, showing adequate perfusion in as little as 1 week, leading to minimization of the time interval between the 2 stages. ${ }^{12}$ No previous studies have been conducted to assess melolabial flap perfusion for nasal reconstruction. Therefore, the objective of this study was to assess melolabial flap neovascularization perfusion after reconstruction of nasal defects using ICG angiography.

\section{Key Points}

Question What is the utility of indocyanine green (ICG) angiography for measuring melolabial flap perfusion before flap division in staged reconstruction of nasal defects?

Findings In this case series study of 10 patients, ICG angiography of distal melolabial flap perfusion with a reference point on the cheek demonstrated that perfusion in the distal flap was excellent compared with the reference point both after clamping and after the division of the pedicle. No correlation existed with a greater interval (longer than 15 days) between the first and second stage and flap perfusion, suggesting that this minimum time is adequate to achieve neovascularization of melolabial flaps.

Meaning In a staged nasal reconstruction using melolabial flaps, ICG angiography is effective for quantification of fluorescence dynamics to assess relative neovascularization perfusion.

\section{Methods}

The study was approved by the Stanford University institutional review board, with a waiver of written informed consent owing to the retrospective nature of the study. Patients with nasal defects undergoing melolabial flap reconstruction between January 1, 2015, and April 30, 2018, with flap perfusion assessed by ICG angiography using the SPY Elite Imaging System were included in the study. Patients younger than 18 years and those with iodide hypersensitivity or hepatic impairment were excluded from this study. All patients underwent operations by a single surgeon (senior author, S.P.M) in an ambulatory surgical center.

During the first stage, the flap was designed according to the defect size and site. Indocyanine green angiography was performed to assess perfusion of the melolabial flap after flap placement. At the second stage, the pedicle was temporarily clamped followed by ICG angiography, which was again performed at the end of the procedure after pedicle division and flap inset. For laser-assisted ICG angiography, $2 \mathrm{~mL}$ of ICG (5 mg) were infused followed by a 10-mL saline bolus. A 90-second duration of SPY imaging was performed and recorded.

Using the SPY-Q software, 2 distinct methods were used to assess neovascularization perfusion of the melolabial flap. First, the ratio of distal flap perfusion compared with a reference point in the cheek was measured at 3 different time points: the midpoint of ICG inflow to the flap, at maximum (peak) fluorescence, and the midpoint of ICG outflow from the flap (Figure 1). The mean of these values was also calculated. The most fluorescent region on the cheek (away from a distinct blood vessel) was used as the reference point. The second method assessed the ratio of the flap-to-cheek ingress (arterial inflow) and egress (venous outflow), representing a relative difference in velocity of blood flow between these 2 areas independent of a particular time point (Figure 2). Flap neovascularization perfusion parameters were assessed after clamping the pedicle at the start of the second stage procedure and after pedicle division and flap inset (Figure 3). 

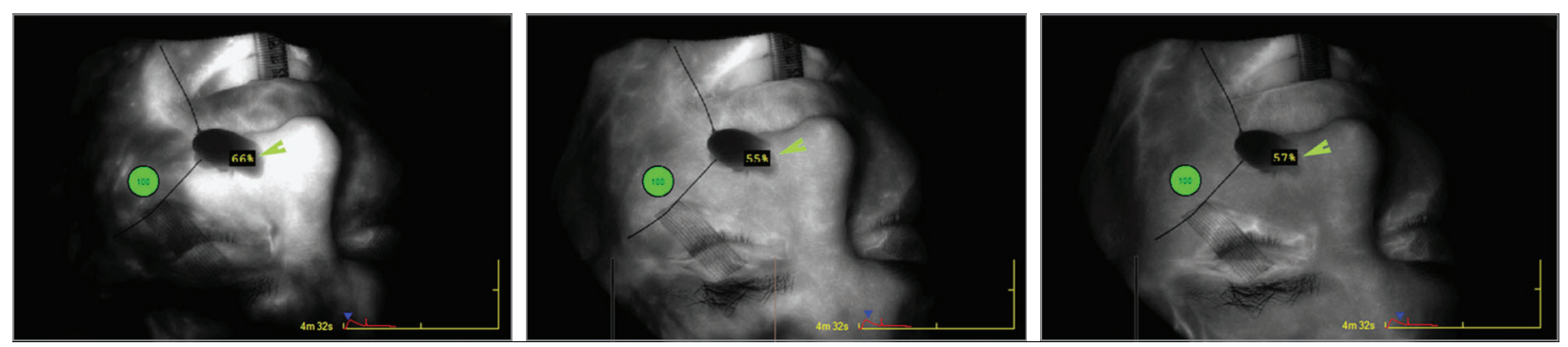

Perfusion percentage of a left melolabial (random) flap after clamping the pedicle in relation to a reference point on the cheek (green circle), during dye indicate flap perfusion percentage measured at the distal flap. Fixed baseline: 15 inflow (A), at peak fluorescence (B), and during outflow (C). Arrowheads

Figure 2. Ingress and Egress Rates (Units/s) of the Flap and Cheek (Time-Independent Method)

A Ingress rate (arterial flow rate)

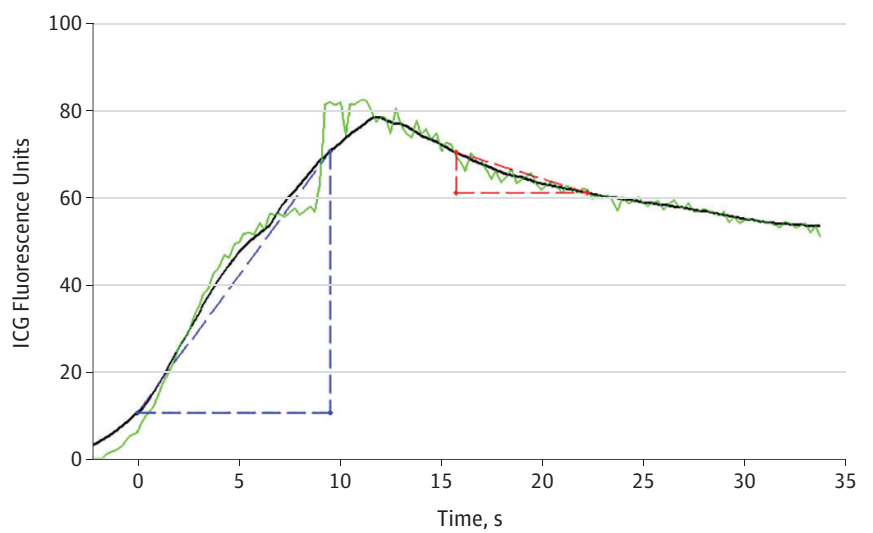

Baseline: 2 units
Ingress: 79 units Ingress rate: 6.3 units/s
C Flap

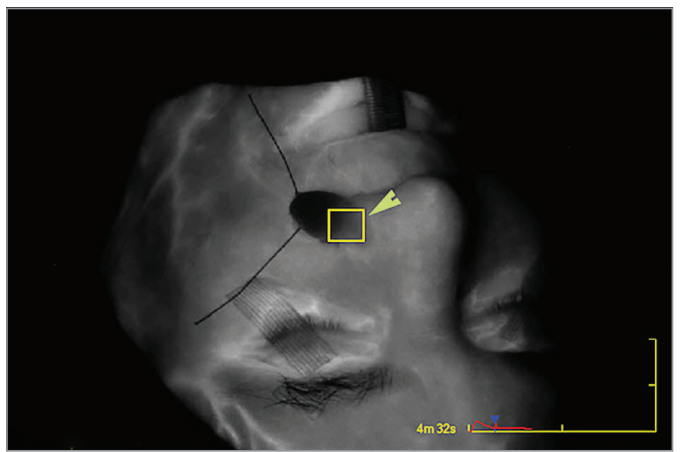

B Egress rate (venous flow rate)

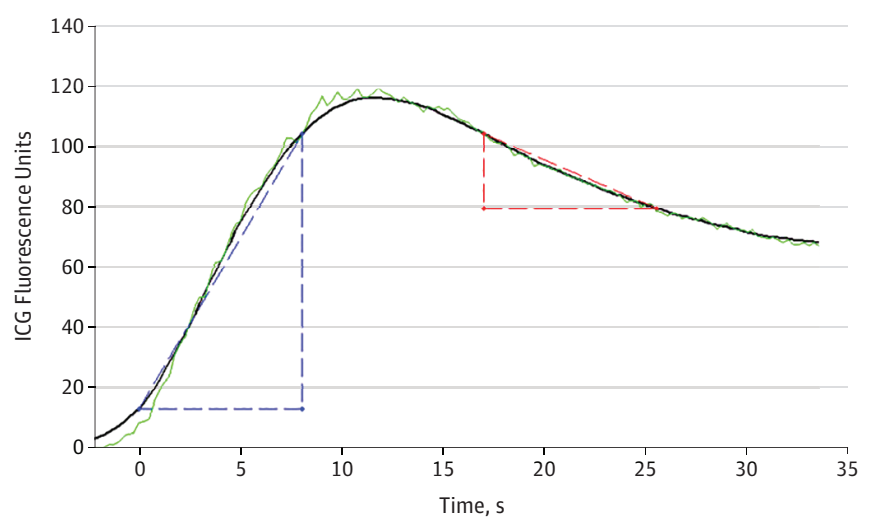

Egress: 28 units

Egress rate: 1.4 units/s

Baseline: 2 units

Ingress: 116 units

Egress: 50 units

Ingress rate: 11.4 units/s

Egress rate: 2.9 units/s

D Cheek

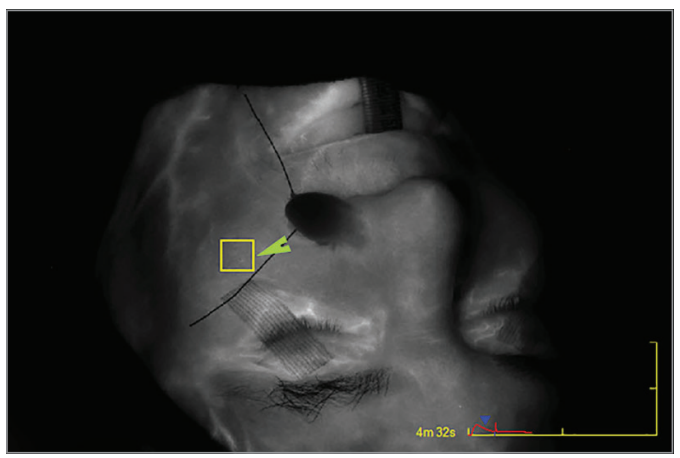

Both values were used to calculate the flap-to-cheek ingress and egress ratio. The graphs on the left show the ingress (arterial flow) rate (A) and egress (venous flow) rate (B) in units/s for the flap and cheek, respectively. The blue triangle corresponds to the ingress rate whereas the red corresponds to the egress rate. Arrowheads indicate the yellow square used to analyze the ingress and egress rates of the flap (C) and cheek (D). Fixed baseline: 15 indicates the background fluorescence value; fps, frames per second; ICG, indocyanine green (units calculated by the SPY-Q software). 
Figure 3. Perfusion Percentage of the Flap After Clamping Versus After Division of the Pedicle During Peak Fluorescence

A Perfusion after pedicle clamping

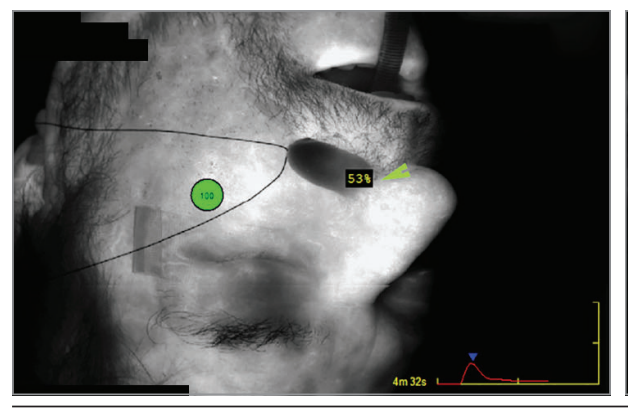

B After division

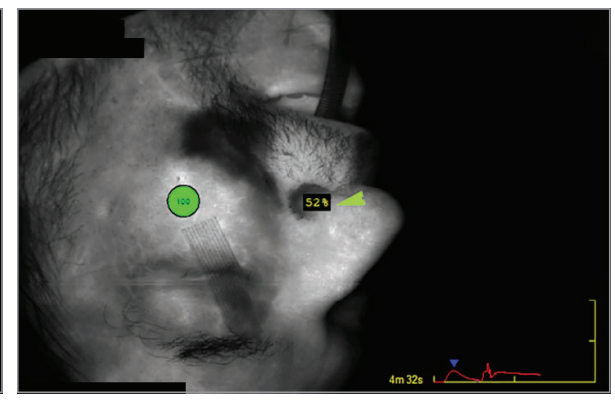

Shows perfusion percentage of the flap in relation to a reference point on the cheek (green circle) after pedicle clamping (A) and after division (B) at peak fluorescence. Arrowheads indicate flap perfusion percentage measured at the distal flap. Fixed baseline: 15 indicates the background fluorescence value; fps, frames per second.

\begin{tabular}{ll}
\hline \multicolumn{2}{l}{ Table 1. Patient and Procedural Characteristics } \\
\hline Characteristic & Total, No. (\%) \\
\hline $\begin{array}{l}\text { Sex } \\
\text { Male }\end{array}$ & $5(50)$ \\
\hline Female & $5(50)$ \\
\hline Age, mean (SD), y & \\
\hline Male & $69(8)$ \\
\hline Female & $63(5)$ \\
\hline Tobacco use & $4(40)$ \\
\hline Diabetes & $1(10)$ \\
\hline Flap division, mean (SD), d & $23(4)$ \\
\hline
\end{tabular}

Standard descriptive statistics were calculated for the patient population as well as perfusion parameters using SPSS (version 25.0, IBM Corp). Linear regressions were used to assess associations of age, sex, time interval between the 2 stages, smoking, diabetes, and radiation history with each perfusion parameter. A paired $t$ test was conducted to assess differences in perfusion parameters after clamping the pedicle and after pedicle division.

\section{Results}

Of 12 patients identified with intraoperative perfusion mapping of their melolabial flap by ICG angiography using the SPY Elite Imaging System, 2 patients were excluded because recordings were not analyzable owing to movement that occurred during intraoperative recording. Therefore, 10 patients were included with at least 3 available SPY imaging recordings for each: after flap inset (first stage), after clamping the pedicle prior to division (second stage), and after pedicle division and flap inset. Table 1 details baseline characteristics of the patients. An equal number of male and female patients were included, and the mean (SD) age was 65.9 (7.5) years. Comorbidities included tobacco use in 4 patients, diabetes in 1 patient, and no patients underwent facial radiation therapy preoperatively. All procedures (first and second stages) and postoperative follow-up periods were uneventful, with no major complications including bleeding events, infections, unplanned admissions, or emergency department visits, and no complete or partial incidences of flap necrosis or wound dehiscence. There were no complications related to the ICG injection.

The time intervals between the first and the second stages were variable ranging from 19 to 30 days (mean [SD], 23[4] days). Retrograde filling of the flap was observed from the recipient site toward the ligated pedicle in all patients. Perfusion parameters measured include ratio of distal flap perfusion compared with a reference point in the cheek at the midpoint of ICG inflow to the flap, at maximum (peak) fluorescence, and the midpoint of ICG outflow from the flap, and the mean captured over these 3 different time points. Flapto-cheek ingress and egress ratios were also measured. Table 2 shows these perfusion parameters after pedicle clamping and after pedicle division. As seen by all perfusion parameters, independent neovascularization of the flap occurred at high levels compared with the cheek, with various mean perfusion ratios ranging from 0.76 to 1.1 after clamping and 0.65 to 1.0 after pedicle division. Flap-to-cheek perfusion ratio was lowest at the midpoint of ICG inflow to the flap and greatest during outflow, signifying both slower arterial filling and venous outflow of the flap compared with the cheek. This finding of slower blood flow to and from the flap compared with the cheek was similarly represented by the ingress and egress ratios that were less than 1.

When correlating the number of days between first and second surgery with flap perfusion, no correlations existed across all perfusion parameters after linear regression, nor did correlations exist with age, sex, tobacco use, or diabetes. A paired $t$ test was conducted between the postclamping and postdivision perfusion parameters to assess the utility of ICG after clamping to accurately predict the postdivision flap perfusion, also shown in Table 2 . The peak and mean (SD) flap-tocheek perfusion ratios showed statistically significant differences postclamping and postdivision, (0.81 [0.2] and 0.77 [0.2]; 95\% CI, 0.005-0.080; $P=.02$; 0.89 and 0.81; 95\% CI, 0.04$0.13 ; P=.001$, respectively).

\section{Discussion}

This study shows the utility of intraoperative assessment of melolabial flap perfusion using laser-assisted ICG angiography 


\begin{tabular}{|c|c|c|c|}
\hline \multirow[b]{2}{*}{ Perfusion Variables } & \multicolumn{2}{|l|}{ Mean (SD) } & \multirow[b]{2}{*}{$P$ Value } \\
\hline & After Clamping & After Division & \\
\hline \multicolumn{4}{|l|}{ Flap-to-cheek } \\
\hline Ingress ratio & $0.75(0.20)$ & $0.71(0.21)$ & .20 \\
\hline Egress ratio & $0.88(0.71)$ & $0.62(0.36)$ & .30 \\
\hline Inflow perfusion ratio & $0.76(0.52)$ & $0.66(0.48)$ & .08 \\
\hline Peak perfusion ratio & $0.81(0.21)$ & $0.77(0.23)$ & .03 \\
\hline Outflow perfusion ratio & $1.10(0.30)$ & $1.00(0.25)$ & .14 \\
\hline Mean flap-to-cheek perfusion ratio & $0.89(0.22)$ & $0.81(0.19)$ & .001 \\
\hline
\end{tabular}

with the associated SPY-Q software providing quantification of fluorescence dynamics data prior to pedicle division. To our knowledge, this is the first study providing such measurements of random flap perfusion used for nasal reconstruction. The ICG angiography measurements showed that perfusion in the distal flap was excellent compared with the cheek reference point both after clamping and after division of the pedicle. In addition, though the sample size was small, no correlation existed with greater interval (longer than 15 days) between the first and second stage and flap perfusion, suggesting that this minimum time is adequate to achieve neovascularization of melolabial flaps. However, more data are required to fully assess this and other factors affecting flap perfusion.

Staged procedure for nasal subunit reconstruction using interpolated flaps is mandatory for providing adequate time for flap neovascularization and survival. Yet, the time interval between these stages has a negative effect on the patient's quality of life and productivity. ${ }^{13}$ By assessing adequate perfusion prior to division, the time interval between stages can be minimized with less risk of flap loss. Neovascularization as early as 1 week has been shown for forehead flaps using ICG angiography, and this same application may be used for melolabial flaps as well. ${ }^{12}$

All perfusion parameters showed independent neovascularization perfusion of the flap at high levels (average, $89 \%)$ compared with the cheek reference. Table 2 demonstrates all perfusion parameters used as a ratio to the cheek. The flap-to-cheek perfusion at peak fluorescence as well as the mean of the 3 time points showed a significant difference after clamping compared with after division. Although these differences are of questionable clinical significance because their mean values were relatively similar (0.81 and $0.77 ; P=.02 ; 0.89$ and $0.81 ; P=.001$; respectively), modifications such as thinning, or refinement of the flap can affect retrograde neovascularization from the bed or surrounding edges of the flap when comparing the postclamping with the postdivision perfusion parameters. Although not statistically significant, ingress and egress ratios also decreased, signifying slower blood flow to and from the flap after refinement as well. Overall, the perfusion ratios obtained after pedicle division were still high, showing good flap perfusion, which corresponds to the absence of any vascular complications in this patient population (Figure 3).

Further interpretation of the data in Table 2 shows a higher ratio of flap-to-cheek perfusion during outflow compared with the inflow and peak values. This shows the presence of some degree of venous congestion of the newly transferred tissue, as well as the slower arterial filling of the flap compared with the cheek. This finding is also supported by the slower ingress and egress ratios of the flap in relation to the cheek.

The evolution in intraoperative perfusion technology has been targeted to minimize the risk and comorbidities of plastic and reconstructive surgery using noninvasive and invasive techniques. ${ }^{20}$ Laser-assisted ICG angiography is 1 technology gaining popularity for its safety as well as high sensitivity and specificity. The associated SPY-Q software is also an advantage of this technology by providing surgeons with the ability to calculate fluorescence dynamics to assess tissue perfusion. It can also be used to assess the arterial inflow and the venous outflow of a particular region in the body or flap. Indocyanine green angiography has been shown to accurately detect differences in intraoperative perfusion. ${ }^{21}$ Clinical studies have described the use of laser-assisted ICG in measuring cardiac output, hepatic function, and retinal and choroidal blood flow analysis. ${ }^{22-25}$ The SPY Elite Imaging System was introduced to assess free flap perfusion, ${ }^{26}$ anastomotic patency, and tissue perfusion in autologous tissue transfer. ${ }^{27}$

In previous studies of interpolated flaps for nasal reconstruction, rates of partial or complete loss have been reported to be between $1 \%$ to $2.9 \%{ }^{28,29}$ Regarding melolabial flaps specifically, the percentage was between 1.2 to $17 \% .{ }^{29-31}$ In this series, all patients included had a perfusion assessment of their flaps. No incidents of partial or complete flap necrosis were reported. Other complications attributed to vascular compromise, such as epidermolysis, infection, or dehiscence, were also not encountered. Thus, as shown by the ICG measurements, the time interval used before the division of the pedicle was sufficient to provide adequate perfusion to the distal flap.

Although smokers may appear healthy enough to undergo multiple procedures, there is a considerable degree of vascular compromise of their flaps. ${ }^{28}$ In previous studies, complications, in particular flap loss, were observed to be higher in tobacco users. ${ }^{28,30}$ Thus, calculating relative flap perfusion in tobacco users, or other high-risk patients, such as those with diabetes or prior radiation, may provide a promising tool to confirm sufficient perfusion prior to division.

\section{Limitations}

Limitations of this study include the small sample size of patients undergoing melolabial flaps with ICG angiography, 
the variability of the population, and the retrospective nature of the study. Fluorescence dynamics quantification requires that no movement occurs during intraoperative recording to avoid unanalyzable data. Recording time was around 90 seconds for adequate interpretation by the software. Although SPY imaging has been introduced in a multitude of indications owing to its high specificity and sensitivity, ${ }^{32}$ the additional cost is another drawback. Costeffectiveness has been evaluated in previous studies, with recommendations for use in patients at high risk for flap failure. ${ }^{33,34}$ For nasal reconstruction, using the SPY Elite Imaging System proved to be cost effective in minimizing the burden on the patient (society) and the health care system simultaneously by decreasing the time between the first- and second-stage surgeries. ${ }^{13}$ Further studies with larger sample sizes are needed to identify patients at higher risk for decreased perfusion of their flap, as well as assess flap perfusion at decreased time intervals to minimize the negative effects on patients' quality of life and productivity.

\section{Conclusions}

The assessment of melolabial flaps using ICG angiography provides quantification of fluorescence dynamics data regarding distal flap perfusion, reducing the risk of vascular-related complications. Indocyanine green angiography can be of particular value in patients at risk for poor neovascularization. Further studies are needed to evaluate shortening of the interval between the stages of melolabial flap reconstruction and subsequent improvement in the quality of life of these patients.

\section{ARTICLE INFORMATION}

Accepted for Publication: October 28, 2018.

Published Online: February 7, 2019.

doi:10.1001/jamafacial.2018.1874

Author Contributions: Dr Most had full access to all of the data in the study and takes responsibility for the integrity of the data and the accuracy of the data analysis.

Study concept and design: Kandathil, Spataro, Most. Acquisition, analysis, or interpretation of data: All authors.

Drafting of the manuscript: Abdelwahab, Kandathil, Spataro.

Critical revision of the manuscript for important intellectual content: Kandathil, Spataro, Most. Statistical analysis: Kandathil, Spataro.

Administrative, technical, or material support: All authors.

Study supervision: Spataro, Most.

Conflict of Interest Disclosures: None reported.

Disclaimer: Dr Most is Associate Editor of JAMA Facial Plastic Surgery, but he was not involved in any of the decisions regarding review of the manuscript or its acceptance.

\section{REFERENCES}

1. Park SS. Reconstruction of nasal defects larger than 1.5 centimeters in diameter. Laryngoscope. 2000;110(8):1241-1250. doi:10.1097/00005537200008000-00001

2. Burget GC, Walton RL. Optimal use of microvascular free flaps, cartilage grafts, and a paramedian forehead flap for aesthetic reconstruction of the nose and adjacent facial units. Plast Reconstr Surg. 2007;120(5):1171-1207.

3. Driscoll BP, Baker SR. Reconstruction of nasal alar defects. Arch Facial Plast Surg. 2001;3(2):91-99. doi:10.1001/archfaci.3.2.91

4. Cutting C. Critical closing and perfusion pressures in flap survival. Ann Plast Surg. 1982;9(6): 524. doi:10.1097/00000637-198212000-00020

5. Buntic R. Flap and replant perfusion monitoring. Online Atlas Of Microsurgery: An Atlas of Microsurgery Techniques and Principles website. https://www.microsurgery.net/monitoring. Accessed June 05, 2018.
6. Eskiizmir G, Tanyeri Toker G, Ozgur E, Tarhan S, Cengiz Ozyurt B. Hemodynamic Changes in Paramedian Forehead Flap. J Craniofac Surg. 2018 29(1):159-162.

7. VIOPTIX. T.Ox. 2018; http://www.vioptix.com/ products/t-ox/. Accessed June 05, 2018.

8. Colwell AS, Craft RO. Near-infrared spectroscopy in autologous breast reconstruction. Clin Plast Surg. 2011;38(2):301-307. doi:10.1016/j.cps.2011.03.014

9. de Weerd L, Mercer JB, Setså LB. Intraoperative dynamic infrared thermography and free-flap surgery. Ann Plast Surg. 2006;57(3):279-284. doi: 10.1097/01.sap.0000218579.17185.c9

10. Singer R, Lewis CM, Franklin JD, Lynch JB. Fluorescein test for prediction of flap viability during breast reconstructions. Plast Reconstr Surg. 1978;61(3):371-375. doi:10.1097/00006534197803000-00010

11. Hölzle F, Loeffelbein DJ, Nolte D, Wolff KD. Free flap monitoring using simultaneous non-invasive laser Doppler flowmetry and tissue spectrophotometry. J Craniomaxillofac Surg. 2006; 34(1):25-33. doi:10.1016/j.jcms.2005.07.010

12. Woodard CR, Most SP. Intraoperative angiography using laser-assisted indocyanine green imaging to map perfusion of forehead flaps. Arch Facial Plast Surg. 2012;14(4):263-269. doi:10.1001/ archfacial.2011.1540

13. Calloway HE, Moubayed SP, Most SP. Cost-effectiveness of early division of the forehead flap pedicle. JAMA Facial Plast Surg. 2017;19(5):418420. doi:10.1001/jamafacial.2017.0310

14. Holm C, Mayr M, Höfter E, Becker A, Pfeiffer UJ, Mühlbauer W. Intraoperative evaluation of skin-flap viability using laser-induced fluorescence of indocyanine green. Br J Plast Surg. 2002;55(8):635644. doi:10.1054/bjps.2002.3969

15. Muckle TJ. Plasma proteins binding of indocyanine green. Biochem Med. 1976;15(1):17-21. doi:10.1016/0006-2944(76)90069-7

16. Ishihara H, Otomo N, Suzuki A, Takamura K, Tsubo T, Matsuki A. Detection of capillary protein leakage by glucose and indocyanine green dilutions during the early post-burn period. Burns. 1998;24 (6):525-531. doi:10.1016/S0305-4179(98)80004-1 17. Surowitz JB, Most SP. Use of laser-assisted indocyanine green angiography for early division of the forehead flap pedicle. JAMA Facial Plast Surg. 2015;17(3):209-214. doi:10.1001/jamafacial.2015.0171

18. Wang $C Y$, Wang $C H$, Tzeng YS, et al. Intraoperative assessment of the relationship between nipple circulation and incision site in nipple-sparing mastectomy with implant breast reconstruction using the SPY Imaging System. Ann Plast Surg. 2018;80(2S Suppl 1):S59-S65.

19. Salman S, Fattahi T, Fernandes R, Steinberg B. Dynamic analysis of maxillary perfusion during Le Fort I osteotomy using indocyanine green. Int J Oral Maxillofac Surg. 2018;47(10):1311-1315. doi:10.1016/ j.ijom.2018.01.010

20. Phillips BT, Munabi NC, Roeder RA, Ascherman JA, Guo L, Zenn MR. The role of intraoperative perfusion assessment: what is the current state and how can i use it in my practice? Plast Reconstr Surg. 2016;137(2):731-741. doi:10.1097/01.prs. 0000475765.83901 .80

21. Diana M, Agnus V, Halvax P, et al. Intraoperative fluorescence-based enhanced reality laparoscopic real-time imaging to assess bowel perfusion at the anastomotic site in an experimental model. Br J Surg. 2015;102(2):e169-e176. doi:10.1002/bjs.9725

22. Ott P. Hepatic elimination of indocyanine green with special reference to distribution kinetics and the influence of plasma protein binding. Pharmacol Toxicol. 1998;83(suppl 2):1-48. doi:10.1111/j.16000773.1998.tb01945.x

23. Zenn MR. Fluorescent angiography. Clin Plast Surg. 2011;38(2):293-300. doi:10.1016/j.cps.2011.03. 009

24. Wood EH. Diagnostic applications of indicator-dilution technics in congenital heart disease. Circ Res. 1962;10:531-568. doi:10.1161/01. RES.10.3.531

25. Flower RW, Hochheimer BF. A clinical technique and apparatus for simultaneous angiography of the separate retinal and choroidal circulations. Invest Ophthalmol. 1973;12(4):248-261.

26. Newman MI, Samson MC. The application of laser-assisted indocyanine green fluorescent dye angiography in microsurgical breast reconstruction. J Reconstr Microsurg. 2009;25(1):21-26. doi:10. 1055/s-0028-1090617

27. Pestana IA, Coan B, Erdmann D, Marcus J, Levin LS, Zenn MR. Early experience with fluorescent angiography in free-tissue transfer reconstruction. 
Plast Reconstr Surg. 2009;123(4):1239-1244. doi:10. 1097/PRS.0b013e31819e67c1

28. Rohrich RJ, Griffin JR, Ansari M, Beran SJ, Potter JK. Nasal reconstruction-beyond aesthetic subunits: a 15-year review of 1334 cases. Plast Reconstr Surg. 2004;114(6):1405-1416.

29. Newlove T, Cook J. Safety of staged interpolation flaps after Mohs micrographic surgery in an outpatient setting: a single-center experience. Dermatol Surg. 2013;39(11):1671-1682. doi:10.1111/ dsu. 12338
30. Thornton JF, Weathers WM. Nasolabial flap for nasal tip reconstruction. Plast Reconstr Surg. 2008; 122(3):775-781. doi:10.1097/PRS.0b013e318182370b

31. Arden RL, Nawroz-Danish M, Yoo GH, Meleca RJ, Burgio DL. Nasal alar reconstruction: a critical analysis using melolabial island and paramedian forehead flaps. Laryngoscope. 1999;109(3):376-382. doi:10.1097/00005537-199903000-00007

32. Pestana IA, Zenn MR. Correlation between abdominal perforator vessels identified with preoperative CT angiography and intraoperative fluorescent angiography in the microsurgical breast reconstruction patient. Ann Plast Surg. 2014;72(6): S144-S149. doi:10.1097/SAP.0000000000000104
33. Wu C, Kim S, Halvorson EG. Laser-assisted indocyanine green angiography: a critical appraisal. Ann Plast Surg. 2013;70(5):613-619. doi:10.1097/ SAP.Ob013e31827565f3

34. Chatterjee A, Krishnan NM, Van Vliet MM, Powell SG, Rosen JM, Ridgway EB. A comparison of free autologous breast reconstruction with and without the use of laser-assisted indocyanine green angiography: a cost-effectiveness analysis. Plast Reconstr Surg. 2013;131(5):693e-701e. doi:10.1097/ PRS.0b013e31828659f4 